\title{
Frequency-Shaped Sliding Mode Control for Flying Height of Pickup Head in Near-Field Optical Disk Drives
}

\author{
W. C. Wu and T. S. Liu
}

\begin{abstract}
To achieve stable flying height of a pickup head in near-field optical disk drives, this study investigates a discrete sliding mode control with a frequency-shaped sliding surface. A compensator is introduced in a sliding mode using a frequency-shaped performance index with linear quadratic optimal and sliding mode control. As an actuator, a piezoelectric bender is used in this study to complement an air bearing at the head/disk interface. Experiments are carried out to demonstrate that the proposed controller performs better in flying height control than an optimal sliding mode controller. Accordingly, the controller can achieve stable flying height control in the presence of certain frequency vibration of optical disks.
\end{abstract}

Index Terms-Air bearing, disk vibration, flying height control, sliding mode control.

\section{INTRODUCTION}

$\mathbf{N}$ EAR-FIELD optical disk drives applying near-field optics can reduce light spot sizes to access finer data tracks and accomplish high data recording density [1]. It adopts a near-field optical pickup head that has to maintain the flying height between pickup head and disk within a near-field focusing length [2]. The recording density can thus be increased by means of the optical resolution improvement. In addition, since optical disks are generally made of a plastic-based material due to its low cost, optical disk vibration amplitude will be severe. Therefore, the passive air bearing flying height mechanism hardly meets the focusing performance requirement in the presence of severe disk vibration. This study attaches a multilayer piezoelectric bender (PZT) to the suspension arm to serve as flying height actuator dealing with the severe disk vibration. Similar designs have been used widely in hard disk drives to achieve head-disk spacing control and fine track following control [3].

The sliding mode control is popular with its robust and insensitive properties to matched disturbance and model uncertainty [4]. In order to deal with certain frequency dynamics, such as high-frequency unmodeled system dynamics or certain frequency disturbances, a frequency shaping approach introduces frequency-dependent weighting matrices in a linear quadratic regulator (LQR) design procedure [5]. Based on the frequency shaping approach, certain frequency compensator dynamics can

Manuscript received August 27, 2004. This work was supported by the National Science Council, Taiwan, R.O.C., under Grant NSC93-2752-E009009-PAE.

The authors are with the Department of Mechanical Engineering, National Chiao Tung University, Hsinchu 30010, Taiwan, R.O.C. (e-mail: wcwu.me89g@nctu. edu.tw; tsliu@mail.nctu.edu.tw).

Digital Object Identifier 10.1109/TMAG.2004.842020

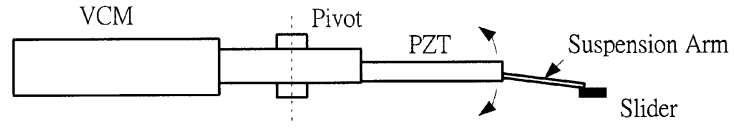

Fig. 1. Flying head with an embedded PZT and a slider.

be introduced into sliding modes through a frequency-shaped sliding surface that is interpreted by linear operators [6]. Certain frequency component dynamics are penalized so as to eliminate undesired high-frequency dynamics or reject certain frequency disturbance.

This study presents a discrete frequency-shaped sliding mode control law for flying height control of near-field optical disk drives. Frequency shaping is conducted such that filtered state variables are penalized at the disk vibration frequency using inverse notch filters in LQR weighting functions. Hence, the controller can achieve stable flying height in the presence of certain frequency vibration of optical disks. To validate the proposed controller, this study carries out experiments for flying height control of a pickup head to demonstrate the effectiveness of the proposed method in comparison with an optimal sliding mode controller.

\section{Plant MODEL}

Fig. 1 depicts a PZT bender and a slider affixed to a flexible suspension arm. The PZT serves as the actuator and the air bearing serves as the passive one to maintain a stable flying height. By using a laser Doppler vibrometer in system identification, a transfer function that relates the PZT voltage $u$ to the tip vertical displacement $y$ of the suspension arm is identified as

$$
\frac{Y(s)}{U(s)}=\frac{K_{P}\left(s^{2}+2 \zeta_{\mathrm{ar}} \omega_{\mathrm{ar}} s+\omega_{\mathrm{ar}}^{2}\right)}{\left(s^{2}+2 \zeta_{r 1} \omega_{r 1} s+\omega_{r 1}^{2}\right)\left(s^{2}+2 \zeta_{r 2} \omega_{r 2} s+\omega_{r 2}^{2}\right)}
$$

where the gain $K_{P}=4.46 \times 10^{8}$, damping ratios $\zeta_{r 1}=0.032, \zeta_{r 2}=0.026$, and $\zeta_{\text {ar }}=0.003$, resonant frequencies $\omega_{r 1}=302 \mathrm{~Hz}$ and $\omega_{r 2}=693 \mathrm{~Hz}$, and anti-resonant frequency $\omega_{\mathrm{ar}}=390 \mathrm{~Hz}$.

The dominant disturbance source is the disk vibration, which comes from the disk deformation, flutter, and spindle motor bias. In order to maintain a constant flying height between the vibratory optical disk and the optical flying head, the flying height control can be treated as a trajectory tracking dealing with a disk vibration waveform, so as to make the flying head height $y$ equal to $y_{H}$, the sum of the disk vibration amplitude $y_{D}$ and a constant flying height $y_{F}$, i.e., the focusing depth for a near-field 
optical disk. Hence, the flying height error $e_{H}$ is defined as $e_{H}=y-y_{H}$.

\section{DisCRETE FREQUENCY-ShAPED SLIDING MODE CONTROL}

A discrete time state-space description of the plant (1) can be written as

$$
\begin{aligned}
\boldsymbol{X}_{1}(k+1) & =\boldsymbol{A}_{11} \boldsymbol{X}_{1}(k)+\boldsymbol{A}_{12} \boldsymbol{X}_{2}(k) \\
\boldsymbol{X}_{2}(k+1) & =\boldsymbol{A}_{21} \boldsymbol{X}_{1}(k)+\boldsymbol{A}_{22} \boldsymbol{X}_{2}(k)+\boldsymbol{B}_{2} u(k) \\
y & =C_{2} \boldsymbol{X}_{2}(k) .
\end{aligned}
$$

\section{A. Optimal Sliding Mode Control}

According to optimal sliding mode control (OSMC) [4], a cost function can be defined as

$$
J_{\mathrm{os}}=\frac{1}{2} \sum_{k=0}^{N-1}\left[\boldsymbol{X}_{1}^{\mathrm{T}} \boldsymbol{Q}_{11} \boldsymbol{X}_{1}+\boldsymbol{X}_{2}^{\mathrm{T}} \boldsymbol{Q}_{22} \boldsymbol{X}_{2}\right] .
$$

The optimal sliding function $S_{\text {os }}$ can thus be defined as

$$
S_{\mathrm{os}}=\boldsymbol{C}_{2} \boldsymbol{K}_{\mathrm{os}} \boldsymbol{X}_{1}+\boldsymbol{C}_{2} \boldsymbol{X}_{2}
$$

where $\boldsymbol{K}_{\mathrm{os}}=Q_{22}^{-1} A_{12}^{\mathrm{T}} \boldsymbol{P}$ comes from the optimal solution of the associated discrete Riccati equation with respect to (5).

In order to design a discrete sliding mode controller, a discrete reaching law approach [7] is adopted as

$$
S_{\mathrm{os}}(k+1)=(1-q h) S_{\mathrm{os}}(k)-\varepsilon h \operatorname{sgn}\left[S_{\mathrm{os}}(k)\right]
$$

where $q>0, \varepsilon>0,1-q h>0, h$ is the sampling period, and $\operatorname{sgn}\left(S_{\mathrm{OS}}\right)$ is a signum function. With a modified sliding function $S_{\text {osr }}=\boldsymbol{C}_{\mathrm{os}}\left(\boldsymbol{X}-\boldsymbol{N}_{x} y_{H}\right)$ based on (6) and the reaching law (7), the control input integrating the reference input $y_{H}$ can be derived as

$$
u_{\mathrm{osr}}=-\boldsymbol{K}_{x} \boldsymbol{X}_{r}+K_{s} S_{\mathrm{osr}}-K_{\mathrm{sgn} \operatorname{sgn}}\left[S_{\mathrm{osr}}\right]+N_{u} y_{H} .
$$

\section{B. Frequency-Shaped Sliding Mode Control}

Frequency-shaped sliding mode control (FSSMC) design, based on ideas of frequency-shaped cost function and optimal sliding surface, is achieved by formulating an optimal LQR problem with frequency-dependent weighting terms [6].

For the cost function in (5), both state variables have fixed weightings for all frequencies. In order to introduce a frequencydependent compensator into the sliding mode, the cost function in (5) is rewritten in the frequency domain by using Parseval's theorem with frequency-dependent weighting functions as

$$
\begin{aligned}
J_{\mathrm{fs}}= & \frac{1}{2 \pi} \int_{-\infty}^{\infty}\left[\boldsymbol{X}_{1}^{*}(\omega) \boldsymbol{Q}_{11}(\omega) \boldsymbol{X}_{1}(\omega)\right. \\
& \left.+\boldsymbol{X}_{2}^{*}(\omega) \boldsymbol{Q}_{22}(\omega) \boldsymbol{X}_{2}(\omega)\right] d \omega \\
= & \frac{1}{2 \pi} \int_{-\infty}^{\infty}\left[\left(\boldsymbol{W}_{1}(\omega) \boldsymbol{X}_{1}(\omega)\right)^{*} \boldsymbol{W}_{1}(\omega) \boldsymbol{X}_{1}(\omega)\right. \\
& \left.+\left(\boldsymbol{W}_{2}(\omega) \boldsymbol{X}_{2}(\omega)\right)^{*} \boldsymbol{W}_{2}(\omega) \boldsymbol{X}_{2}(\omega)\right] d \omega \\
= & \frac{1}{2 \pi} \int_{-\infty}^{\infty}\left[\eta_{1}^{*} \eta_{1}+\eta_{2}^{*} \eta_{2}\right] d \omega
\end{aligned}
$$

where the notation ()$^{*}$ denotes a complex conjugate transpose, $\boldsymbol{W}_{i}$ is the spectral factor of $\boldsymbol{Q}_{\boldsymbol{i}}$, i.e., $\boldsymbol{Q}_{i}(\omega)=$
$\boldsymbol{W}_{i}^{*}(\omega) \boldsymbol{W}_{i}(\omega), \eta_{i}(\omega)=\boldsymbol{W}_{i}(\omega) \boldsymbol{X}_{i}(\omega)$ are outputs of each filter with transfer functions $\boldsymbol{W}_{i}(\omega)$ and inputs $\boldsymbol{X}_{i}(\omega)$. In order to obtain a casual optimal solution that minimizes the cost function, both weighting functions $\boldsymbol{Q}_{i}(\omega)$ are assumed to be proper rational functions of squared frequency $\omega^{2}$ [5]. According to (9), if $\boldsymbol{W}_{i}$ represents a bandpass filter, the minimization of $J_{\mathrm{fs}}$ will penalize the component of $\boldsymbol{X}_{i}(\omega)$ in its frequency band [6].

Considering the plant output dynamics in (4), it is feasible to perform frequency shaping for $\boldsymbol{X}_{2}$ instead of $y$ to achieve required control performance. Hence, $\boldsymbol{W}_{1}(\omega)$ can be treated as constant for any frequency and $\boldsymbol{W}_{2}(\omega)$ can thus be designed as a required compensator expressed as

$$
\begin{aligned}
\boldsymbol{X}_{w}(k+1) & =\boldsymbol{A}_{w} \boldsymbol{X}_{w}(k)+\boldsymbol{B}_{w} \boldsymbol{X}_{2}(k) \\
\eta_{2}(k) & =\boldsymbol{C}_{w} \boldsymbol{X}_{w}(k)+\boldsymbol{D}_{w} \boldsymbol{X}_{2}(k) .
\end{aligned}
$$

The sliding mode synthesis of the required compensator and sliding function can be transformed into the discrete LQR procedure. Integrating the compensator state dynamics (10) into the system (2) yields an augmented system written as

$$
\boldsymbol{X}_{e}(k+1)=\boldsymbol{A}_{e} \boldsymbol{X}_{e}(k)+\boldsymbol{B}_{e} \boldsymbol{X}_{2}(k)
$$

where $\boldsymbol{X}_{e}(k)=\left[\begin{array}{ll}\boldsymbol{X}_{w}(k) \boldsymbol{X}_{1}(k)\end{array}\right]^{\mathrm{T}}, \boldsymbol{A}_{e}=\left[\begin{array}{cc}\boldsymbol{A}_{w} & 0 \\ 0 & \boldsymbol{A}_{11}\end{array}\right]$, and $\boldsymbol{B}_{e}=$ $\left[\begin{array}{ll}\boldsymbol{B}_{w} & \boldsymbol{A}_{12}\end{array}\right]^{T}$. Thus, the cost function for the system dynamics (11) is written as

$$
J_{\mathrm{fs}}=\frac{1}{2} \sum_{k=0}^{N-1}\left[X_{e}^{\mathrm{T}} Q_{e} X_{e}+2 X_{e}^{\mathrm{T}} N_{e} X_{2}+X_{2}^{\mathrm{T}} R_{e} X_{2}\right] .
$$

Accordingly, the optimal solution of (12) can be solved as

$$
X_{2}(k)=-K_{w} X_{w}(k)-K_{1} X_{1}(k) .
$$

Hence, the sliding function for FSSMC can be designed as

$$
S_{\mathrm{fs}}(k)=\boldsymbol{C}_{2} \boldsymbol{K}_{w} \boldsymbol{X}_{w}(k)+\boldsymbol{C}_{2} \boldsymbol{K}_{1} \boldsymbol{X}_{1}(k)+\boldsymbol{C}_{2} \boldsymbol{X}_{2}(k) .
$$

The sliding function in (14) is no longer a hypersurface in the original state space. Instead, it is a linear operation on state variables [5].

With the reaching law and a modified sliding function $S_{\mathrm{fsr}}=$ $\boldsymbol{K}_{w} \boldsymbol{X}_{w}+\boldsymbol{K}_{1}\left(\boldsymbol{X}_{1}-\boldsymbol{N}_{x 1} y_{H}\right)+\left(\boldsymbol{X}_{2}-\boldsymbol{N}_{x 2} y_{H}\right)$, the control input incorporating the reference input $y_{H}$ can thus be derived as

$$
\begin{array}{r}
u_{\mathrm{fsr}}(k)=-\boldsymbol{K}_{u w} \boldsymbol{X}_{w}(k)-\boldsymbol{K}_{u x} \boldsymbol{X}_{r}(k)+K_{u s} S_{\mathrm{fsr}}(k) \\
-K_{u \operatorname{sgn} \operatorname{sgn}}\left[S_{\mathrm{fsr}}(k)\right]+N_{u} y_{H}(k) .
\end{array}
$$

Since state variables may not be all measurable in practice, state observers can be adopted to accomplish state feedback. Fig. 2 shows a block diagram of the FSSMC accompanied with a state observer.

\section{EXPERIMENTAL RESULTS AND CONCLUSION}

The experimental setup consists of a PZT bender of Model no. PL122.251 made by Physik Instrumente, a corresponding PZT driver, two laser Doppler interferometers, a PCI-1716 multifunction card, and a personal computer to perform a real-time PC-based control experiment at a sampling rate of $10 \mathrm{kHz}$. 


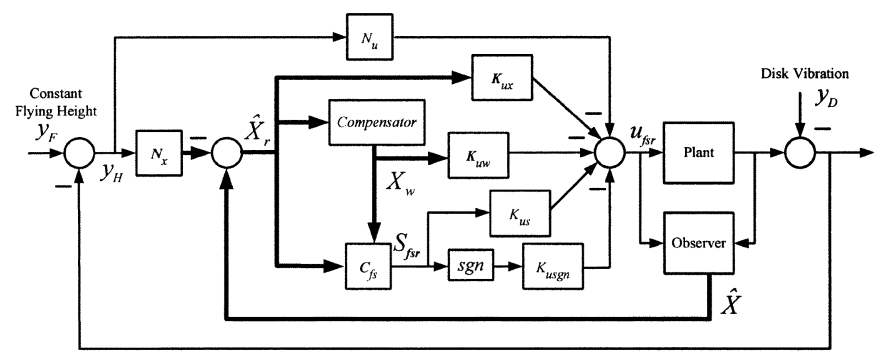

Fig. 2. Block diagram of FSSMC.
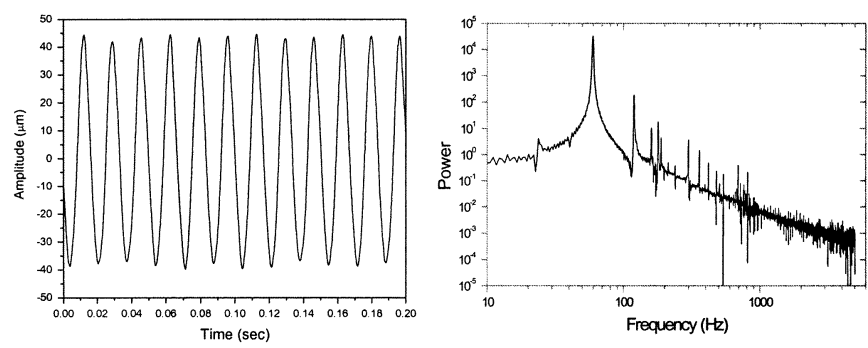

Fig. 3. Waveform and power spectrum of disk vibration.

Fig. 3 depicts a vibration waveform and power spectrum of a polycarbonate substrate optical disk at a constant speed of $3600 \mathrm{rpm}$. The dominant frequency of the vibration waveform is about $60 \mathrm{~Hz}$ synchronous with the spindle motor speed. Hence, the compensator in (10) is designed based on an inverse notch filter of the form

$$
W_{N}(\omega)=\frac{s^{2}+\omega_{N} s+\omega_{N}^{2}}{s^{2}+\omega_{N}^{2}}
$$

where the notch frequency $\omega_{N}=2 \pi \times 60 \mathrm{~Hz}$. The frequency content of the optimal sliding mode is shaped by using a frequency-dependent compensator presented in (10), such that a higher gain can be obtained at the designated $\omega_{N}$. With sufficient frequency separation of filter dynamics around $\omega_{N}$ and
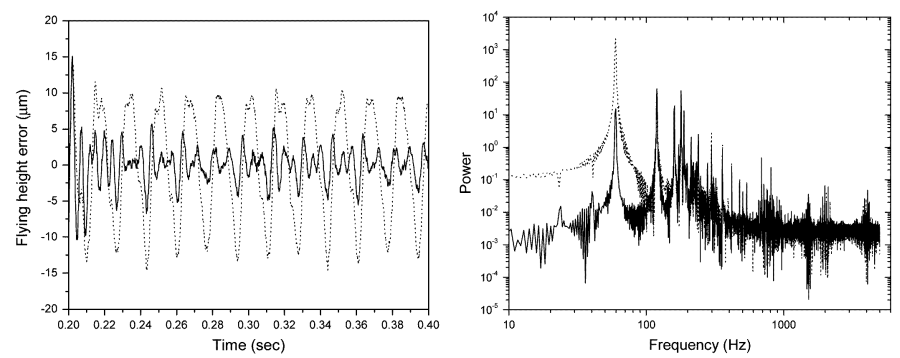

Fig. 4. Amplitudes and power spectrums of flying height error for OSMC (dotted line) and FSSMC (solid line).

significant plant dynamics around $\omega_{r 1}, \omega_{r 2}$, and $\omega_{\mathrm{ar}}$, this compensation hardly affects system dynamics away from the prescribed frequency $\omega_{N}$.

Experimental results compare both controllers of OSMC in (8) and FSSMC in (15) as shown in Fig. 4. The flying height error of FSSMC is smaller than that of OSMC. Power spectrums comparison also shows that FSSMC yields smaller error than OSMC. The remaining flying height error of FSSMC mainly comes from modeling inaccuracy, PZT drift, etc.

\section{REFERENCES}

[1] T. D. Milster, "Near-field optics: A new tool for data storage," Proc. IEEE, vol. 88, no. 9, pp. 1480-1490, Sep. 2000.

[2] K. Ito, H. Saga, H. Nemoto, and H. Sukeda, "Advanced recording method using a near-field optics and the GMR head," in Optical Data Storage Conf. Dig., 2000, pp. 30-32.

[3] M. Kobayashi and R. Horowitz, "Track seek control for hard disk dual-stage servo systems," IEEE Trans. Magn., pt. 1, vol. 37, no. 2, pp. 949-954, Mar. 2001.

[4] V. I. Utkin, Sliding Modes in Control Optimization. New York: Springer-Verlag, 1992.

[5] N. K. Gupta, "Frequency-shaped cost functional: Extension of linearquadratic-Gaussian design methods," J. Guid. Contr., vol. 3, no. 6, pp. 529-535, 1980.

[6] K. D. Young and U. Ozguner, "Frequency shaping compensator design for sliding mode," Int. J. Contr., vol. 57, no. 5, pp. 1005-1019, 1993.

[7] W. Gao, Y. Wang, and A. Homaifa, "Discrete-time variable structure control systems," IEEE Trans. Ind. Electron., vol. 42, no. 2, pp. 117-122, Apr. 1995. 